\title{
MALIGNANT PHYLLODES TUMOR OF THE BREAST: REPORT OF TWO CASES AND A RARE ASSOCIATION
}

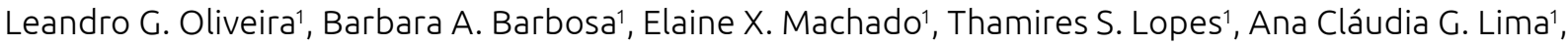
Alexandre M. X. de Jesus'1, Sebastião A. Pinto', Juarez A. de Sousa'

IInstituto Goiano de Oncologia e Hematologia - Goiânia (GO), Brazil.

Introduction: Phyllodes tumors (PT) represent less than $1 \%$ of all breast tumors. Most of them are benign, but up to $30 \%$ show malignant stroma. Malignant PT of the breast (MPTB) are characterized by marked stromal cellularity, atypia and high mitotic rate ( $>$ ou = a 10 per 10 high-power fields). Unlike benign PT, MPTB can be clinically aggressive, with local recurrence rates ranging from 23-30\%. Malignant change of the epithelium in MPTB is rare. The most frequent occurrence is infiltrating carcinoma of various types while ductal carcinoma in situ (DCIS) is more rare. The purpose of this study was to present two cases of MPTB, one of them with a concomitant DCIS. Case reports: Case 1: A 49-year-old female patient presented a history of mass in the right breast with accelerated growth. Core biopsy was compatible with a fusocellular neoplasm. Patient underwent simple mastectomy with surgical margins free. Macroscopy evidenced a 12x11 cm lesion. Immunohistochemistry (IHC) showed a tumor with high mitotic rate (12 mitoses/hpf), ki-67 10\%, CD-34 and vimentin +, S-100 protein and AE1/AE3 ceratines -, compatible with malignant phyllodes tumor. Case 2: A 54-year-old female patient presented with a firm mass in the left breast with accelerated growth. Core biopsy was compatible with a low grade MPTB (6 mitoses/hpf). IHC was positive for CD-3 and vimentin and ki-6715\%. Patient underwent left adenomastectomy with sentinel node biopsy. The features were consistent with MPTB of $4.5 \mathrm{x} 3.0 \mathrm{~cm}$. The epitelial component showed a $0.7 \times 0.6 \mathrm{~cm}$ DCIS, nuclear grade 2, solid with hormonal-receptors positives $(>90 \%)$. Surgical margins were free of tumor. Discussion: PT represent a specific subset of breast soft tissue tumors composed of a connective stroma and epithelial elements. These epithelial components may show a range of metaplastic (apocrine, squamous) and proliferative changes. However, DCIS in association with PT is extremely rare, with only few cases reported in the literature. The role of adjuvant radiation or chemotherapy following partial breast surgery (PBS) or mastectomy is still unclear due to the rarity of these tumors. Conclusion: In this study, we described a MPTB that harbored an intraductal carcinoma. This result could determine an absolute indication for adjuvant radiotherapy if the patient underwent PBS. Although the stroma in these tumors is commonly the aggressive component, the epithelial component also requires close and careful histological appraisal. 